\title{
Research on Variable Transmission Ratio and Yaw Rate Control Strategy of Electric Forklift Steering-by-Wire System
}

\author{
Guohua Xiang \\ Institute of Industry \& Equipment Technology, Hefei University of Technology \\ E-mail: guohua_xiang@163.com \\ Benxian Xiao \\ School of Electrical Engineering and Automation, Hefei University of Technology \\ E-mail: xiaobenxian@126.com
}

\begin{abstract}
Combined with the trans mission ratio characteristics of forklift steering-by-wire (SBW) system, through the application of fuzzy control technology, the variable trans mission ratio function is designed based on the steering handle angle and vehicle speed, and simulation analysis of sinusoidal steering is done at low-speed and high-speed. Simulation results show that the fuzzy variable transmission ratio control can make forklift steering light \& sensitive at low-speed and steering steady \& heavy at high speed, also it can improve the operation stability and reduce the driver's load. Discuss the relationship between yaw rate and forklift handling stability, propose the yaw rate feedback control strategy based on the fuzzy variable transmission ratio control, and design a fuzzy self-adaptive PID controller. Simulation results show that the SBW system based on the fuzzy variable trans mission ratio control with yaw rate feedback can accurately and quickly track the desired yaw rate, and reduce or even eliminate the overshoot phenomenon, improve the forklift dynamic performance.
\end{abstract}

Index Terms - Electric forklift, SBW, Variable transmission ratio, Yaw rate feedback, Fuzzy control.

\section{INTRODUCTION}

Electric forklift has the advantages of energy conservation and environmental protection, high reliability, low production cost and easy upgrading and so on. So it greatly meets the world's development needs. The electric forklift with variable cargo need frequently steer, thus the requirements of the forklift's steering system are higher than other vehicles' steering systems. It not only requires steering sensitivity but also high steering stability. The famous forklift companies in the world have already produced a range of electric forklifts including pallet forklifts, reach forklifts, narrow aisle forklifts, ex-proof forklifts and so on. Electric forklifts account for $70 \%$ of the whole forklift products in Europe while they only account for about $20 \%$ in China in 2015. The main factor limiting the development of China's forklift is the back- wardness of steering system's design.

As the steering transmission ratio of the mechanical steering(MS) system, hydraulic power steering(HPS) system, electric hydraulic power steering(EHPS) system, electric power steering(EPS) system and other mechanical or hydraulic steering system is fixed or changes in a small range, electric forklift shows the strong nonlinear time-variant steering characteristics with the changes of the steering handle angle and vehicle speed. When the electric forklift fully loaded with cargo frequently steers, the driver needs to forecast and offset the variable steering characteristics in real time. Undoubtedly it will enhance the driver's mental and physical burden, increasing unsafe factors[1-3]. SBW system can design suitable steering transmission ratio according to the forklift's variable steering handle angle and vehicle speed. Besides it can adjust forklift's steering characteristics dynamically and decrease the driver's manual adjustment frequency. Thus the forklift's operation stability has a great improvement.

SBW system thoroughly breaks traditional mechanical or hydraulic transmission mechanism's design limits, completely removes the mechanical connections between steering wheel and steering execution mechanism[4,5], avoiding the interferences among these mechanisms. Meanwhile SBW system acco mplishes the active steering control, road feeling simulation and steering wheel returnable control through steering motor and road feeling motor. Therefore SBW system has the advantages of variable transmission ratio and adjustable properties.

Combined with the actual steering-by-wire electric forklift, this paper analyzes and studies the ideal transmission ratio factors. We design non-linear variable transmission ratio function based on fuzzy control, and make simulations of different variable transmission ratio functions under different situations. The results show that variable transmission ratio can improve the forklift's steering sensitivity and stability.

According to the influences that the yaw rate has on the forklift operation stability, we propose the yaw rate feedback control strategy based on the fuzzy self-adaptive PID controller, and make simulations and analysis on 
steering-by-wire electric forklift TE60. The model of TE60 is showed in Fig.1.

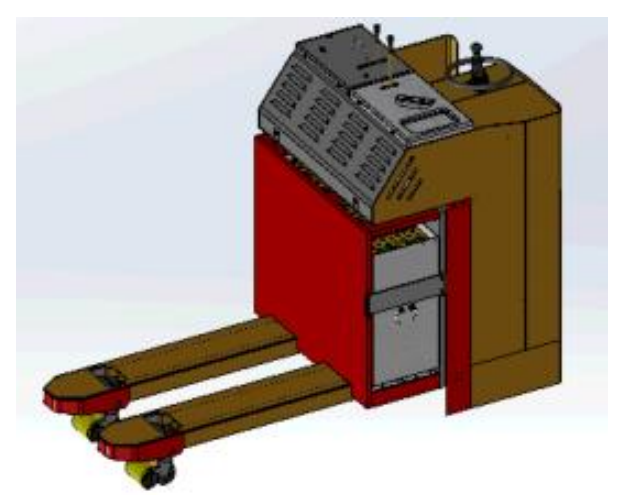

Fig. 1. The model of TE60

The main research work of this paper is listed below:

(1) Introduces the compositions and functions of SBW system.

(2) Explains the concept of ideal transmission ratio. Builds the ideal transmission ratio model by analyzing the factors influencing transmission ratio. Designs the fuzzy variable transmission ratio according to expert experience. Simulation is done to prove the fuzzy variable transmission ration control has a good improvement of the forklift's handling stability.

(3) Based on the fuzzy variable transmission ratio control, designs yaw rate feedback fuzzy self-adaptive PID control. This paper proves that the yaw rate feedback fuzzy self-adaptive PID control have more obvious improvements of the forklift's dynamic characteristic by comparing with several control methods through the simulation analysis.

The rest of this paper is organized into four sections: section II reviews some mean ingful related work. Section III introduces the compositions and functions of SBW system. Section IV is about the research of various transmission ratio control. Section $V$ is the yaw rate feedback fuzzy self-adaptive PID control part.. Section VI concludes our work.

\section{RELATED WORK}

Many scholars have already done some meaningful researches on SBW system. For example, Y. Marumo, M. Nagai and N. Katagiri proposed the state feedback control method to drive the steering angle of the trucks equipped with SBW system to track the reference steering angle[6-8]. While This method's disadvantage is that the closed-loop performance can't be stable when the road conditions change very fast. Y. Yamaguchi, K. Nam, H. Fujimoto, et designed the steering control by using the online estimations of the cornering stiffness and tire slip angle[9,10]. However, the stiffness and tire slip angle can't be accurately estimated under different road conditions. A. E. Cetin, M. A. Adli, and D. E. Barkana designed the adaptive pole placement controller to reduce the tracking error[11]. But this controller didn't consider the road conditions in the parameter.

$\mathrm{H}$. Wang et proposed the sliding mode control method applied in SBW system to improve the vehicle's steering performance against parameter uncertainties and different road condition[12]. Avinash Balachandran and J. Christian Gerdes proposed a steering feel model of appropriate fidelity to capture important elements of steering feel while remaining intuitive to tune objectively with a small set of parameters $[13,14]$. Segawa, et designed lateral acceleration and yaw rate feedback control system with a SBW system and showed more improved driving stability than differential brake control[15]. Yih and Gerdes proposed a method for altering a vehicle's handling characteristics by augmenting the driver's steering command with full vehicle state feedback, which are chassis sideslip angle and yaw rate, by using a combination of global positioning system and inertial navigation system sensors[16].

\section{THE COMPOSITIONS AND FUNCTIONS OF SBW SYSTEM}

SBW system is mainly composed of three parts, namely Steering Handle Module, Steering Execution Module, Electronic Control Unit(ECU).

\section{A. Steering Handle Module}

It is usually composed of steering handle, torque transducer, reduction gear, steering angle senor, current sensor, and road feeling motor, etc. ECU can receive the torque signal and corner signal measured by sensors when the driver turns the steering handle, and realize the steering control by controlling the motor running after analyzing the signals. Meanwhile, it can also accomplish road feeling simulation and steering handle returnable control by controlling the road feeling motor [17-19]. Besides, ECU can realize the steering handle resistance control, the middle line driving control and so on.

\section{B. Steering Execution Module}

This module is mainly composed of rack and pinion steering gear, steering wheel angle sensor, motor reduction gear, steering motor and current sensor, etc. The main function of Steering Execution Module is executing ECU's control commends, realizing the steering control of steering wheel, and feeding the condition signals measured by steering wheel angle sensor and current sens or back to ECU in time. In addition, Steering Execution Module can also accomplish the steering wheel returnable control.

\section{C. $E C U$}

Its main function is collecting signals measured by sensors and making analysis and decisions. Then ECU controls the road feeling motor and steering motor to get corresponding road feeling and angle. So the system can ensure the quick respond and safety under different situations and speeds. 


\section{RESEARCH ON VARIABLE TRANSMISSION RATIO OF THE FORKLIFT'S SBW SYSTEM}

Steering-by-wire (SBW) system's transmission ratio is variable. The reasonable designed transmission ration can solve the contradiction between light and sensitivity in mechanical or hydraulic steering system, realize the ideal steering characters and reduce the driver's load.

The design of SBW system's transmission ratio should meet the following require ments. When the forklift steers at a low speed, the transmission ratio should be designed smaller to ensure the steering flexibility. So the system can get larger steering wheel angle when the driver turns a small hand angle. When the forklift steers at a high speed, the transmission ratio should be designed larger to ensure the steering stability. So the system can get smaller steering wheel angle when the driver turns a large hand angle. When the forklift turns a small corner or steers in the straight line, the larger transmission ratio can get better steering precision. When the forklift turns a big corner, the smaller transmission ratio can ensure the steering portability.

\section{A. The Concept of Ideal Transmission Ratio}

SBW system can make the steering gain perform a fixed ratio relationship not related to the speed by changing the transmission ratio to simplify the steering operation and reduce the driver's load[20,21]. We define this steering transmission ratio as ideal transmission ratio. The transmission ratio $(i)$ can be defined as:

$$
i=\frac{\delta_{s w}}{\delta_{w h}}
$$

$\delta_{s w}$ represents the steering handle angle. $\delta_{w h}$ is the steering wheel angle.

When we study the steering characteristics, the steering gain is a very important factor. The yaw rate gain $\left(G_{\delta_{s w}}^{\omega_{\gamma}}\right)$ can be defined as:

$$
G_{\delta_{s w}}^{\omega_{y}}=\frac{\omega_{\gamma}}{\delta_{s w}}
$$

Where $\omega_{\gamma}$ represents the yaw rate, $\delta_{s w}$ is the steering handle angle.

$G_{\delta_{s w}}^{\omega_{\gamma}}$ is also called steering sensitivity. It refers to the forklift's steady uniform circular motion response when the steering handle has an input of steering angle. It reflects the forklift's sensitivity and responsiveness to the input of the steering handle angle. The yaw rate gain is an important factor to measure the forklift's operation stability.

According to the literature[22-25], we get the model:

$$
G_{\delta_{s w}}^{\omega_{y}}=\frac{\frac{u}{L}}{i\left(1+\frac{m}{L^{2}}\left(\frac{a}{k_{2}}-\frac{b}{k_{1}}\right) u^{2}\right)}
$$

Where $\mathrm{L}$ is the wheelbase. It is a plus b. $u$ is the vehicle speed. $m$ is the forklift's mass. $a$ is the distance between mass center and front-axle. $b$ is the distance between mass center and back-axle. $k_{1}$ is the cornering stiffness of front wheel. $k_{2}$ is the cornering stiffness of back wheel.

Bring (2) into (3), we can get the trans mission ratio:

$$
i=\frac{\frac{u}{L}}{\frac{\omega_{\gamma}}{\delta_{s w}}\left(1+\frac{m}{L^{2}}\left(\frac{a}{k_{2}}-\frac{b}{k_{1}}\right) u^{2}\right)}
$$

Eq.(4) indicates that the transmission ratio is not only related to the design of its mechanical parameters, but also to $\delta_{s w}, u$, and other motion variables.

\section{B. The Method of Confirming The Ideal Transmission Ratio Based on The Model}

The ideal trans mission ratio based on the model is designed according to constant yaw rate gain. When the forklift's speed is under $u_{1}$, the transmission ratio of SBW system should be a fixed value $\left(i_{\min }\right)$ to ensure the steering stability. When the steering handle angle $\left(\delta_{s w}\right)$ is the $\max \left(\delta_{\text {swmax }}\right)$, and the steering wheel angle $\left(\delta_{w h}\right)$ is the $\max \left(\delta_{\text {whmax }}\right)$,we can get (5):

$$
i_{\min }=\frac{\delta_{s w \max }}{\delta_{w h \max }}
$$

When the speed is above $u_{1}$, the yaw rate gain $\left(G_{\delta_{s w}}^{\omega_{\gamma}}\right)$ should retain constant according to the design requirements of ideal transmission ratio. Researches show that the suitable yaw rate gain ranges from 0.25 to 0.52 . We can get SBW system's ideal transmission ratio based on linear two degree of freedom model combined with (3):

$$
i= \begin{cases}i_{\min }, & 0 \leq u<u_{1} \\ \frac{\frac{u}{L}}{G_{\delta_{s w}}^{\omega_{y}}\left(1+\frac{m}{L^{2}}\left(\frac{a}{k_{2}}-\frac{b}{k_{1}}\right) u^{2}\right)}, & u \geq u_{1}\end{cases}
$$

This paper has taken some calculations and simulations according to TE60 forklift's parameters. Specific parameters is showed in Table.1.

Table 1. The model parameters of electric forklift

\begin{tabular}{|c|c|c|}
\hline Parameters & Value & Unit \\
\hline$m$ & 1100 & $\mathrm{~kg}$ \\
\hline$a$ & 0.518 & $\mathrm{~m}$ \\
\hline$b$ & 0.95 & $\mathrm{~m}$ \\
\hline$k_{1}$ & -55856 & $\mathrm{~N} / \mathrm{rad}$ \\
\hline$k_{2}$ & -55856 & $\mathrm{~N} / \mathrm{rad}$ \\
\hline$u_{\max }$ & 15 & $\mathrm{~km} / \mathrm{h}$ \\
\hline$\delta_{s w}$ & {$[-90,90]$} & degree \\
\hline$\delta_{w h}$ & {$[-90,90]$} & degree \\
\hline
\end{tabular}

I.J. Intelligent Systems and Applications, 2016, 10, 21-30 
Combined with (6) and Table 1, we can get the relationship curve between transmission ratio and vehicle speed in different yaw rate gains:

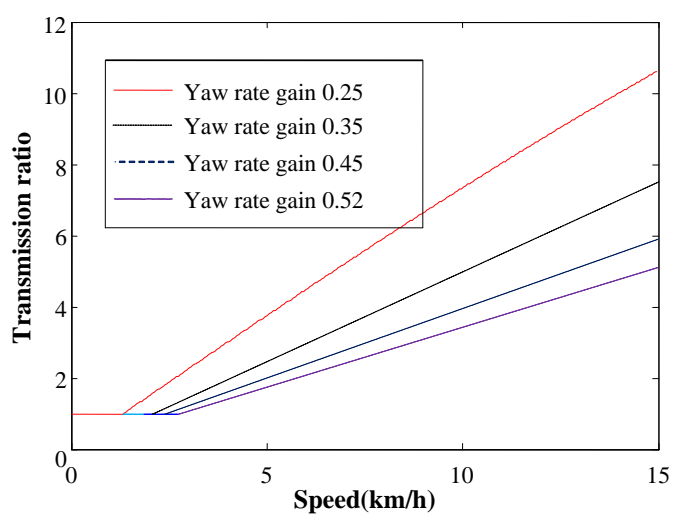

Fig.2. Relationship curve bet ween transmission ratio and vehicle speed in different yaw rate gains

\section{The Design of Fuzzy Variable Transmission Ratio}

Traditional variable transmission ratio control method is mainly built based on mathematical mode1[26,32]. It belongs to static control. But practically when the forklift moves, it can't avoid some problems such as complicated road conditions, mechanical parts and tire deformation and other questions. It is under a dynamic condition. Thus we propose the fuzzy variable transmission ratio control method in this paper.

We can get that transmission ratio is related to many variables according to (4). This paper takes the forklift's $u$ and $\delta_{s w}$ as the fuzzy controller's input variables, and $i$ as output variable.

$u$ ranges from 0 to $15 \mathrm{~km} / \mathrm{h}$. Fuzzy set is $\{\mathrm{NB}, \mathrm{NM}, \mathrm{NS}, \mathrm{Z}, \mathrm{PS}, \mathrm{PM}, \mathrm{PB}\}$. Discourse domain is $\{0,2.5,5,7.5,10,12.5,15\}$. The designed membership function of vehicle speed is showed in Fig.3.

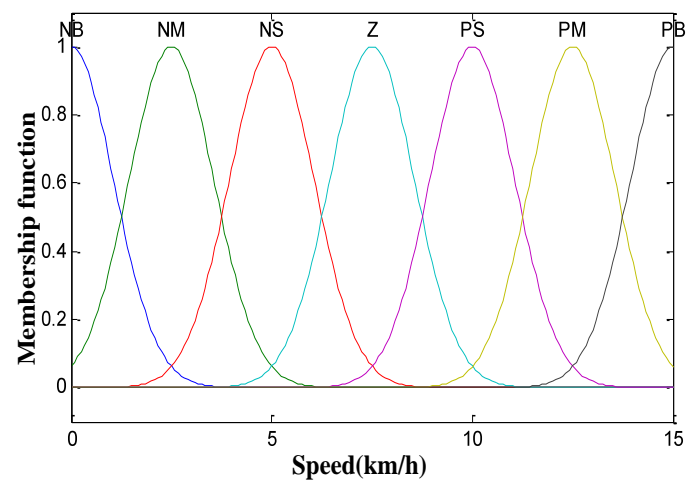

Fig.3. Membership function chart of vehicle speed

$\delta_{s w}$ ranges from $-90^{\circ}$ to $90^{\circ}$. Fuzzy set is \{NB,NM,NS,Z,PS,PM,PB\}. Discourse domain is $\{-90,-60,-30,0,30,60,90\}$. The designed membership function of steering handle angle is showed in Fig.4.

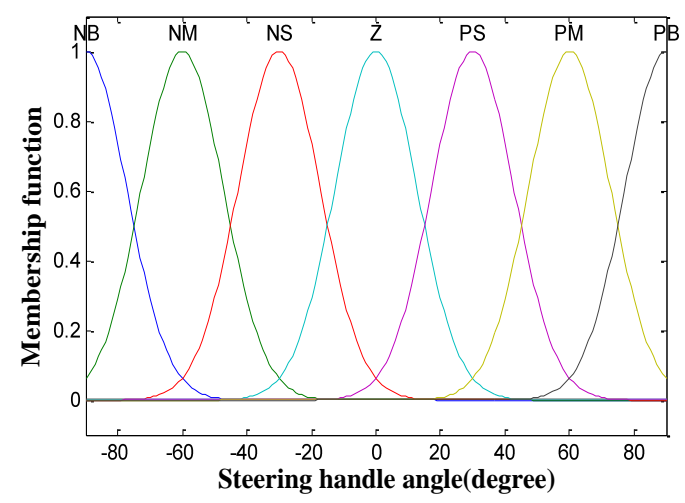

Fig.4. Membership function chart of steering handle angle

$i$ ranges from 1 to 13 . Fuzzy set is $\{\mathrm{NB}, \mathrm{NM}, \mathrm{NS}, \mathrm{Z}$, PS, PM, PB $\}$. Discourse domain is $\{1,3,5,7,9,11,13\}$. The designed membership function of transmission ratio is showed in Fig.5.

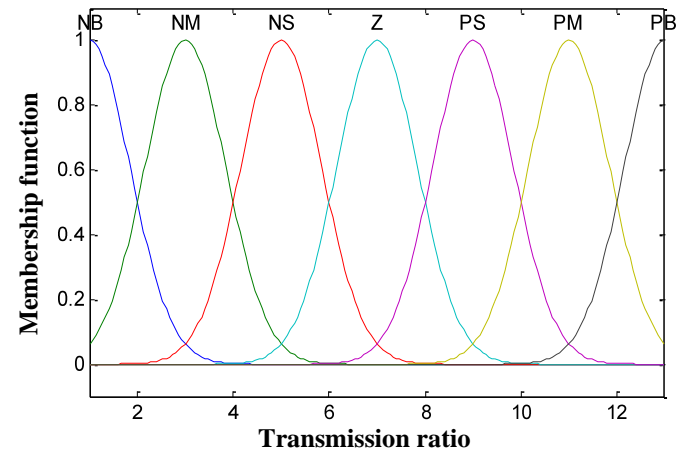

Fig.5. Membership function chart of transmission ratio

According to the drivers' practical experiences and expert knowledge, we make fuzzy variable transmission ratio control rule table:

Table 2 . The control rule table of fuzzy variable transmission ratio

\begin{tabular}{|l|l|l|l|l|l|l|l|}
\hline \multirow{2}{*}{$\delta_{s w}$} & \multicolumn{7}{|c|}{$u$} \\
\cline { 2 - 8 } & NB & NM & NS & Z & PS & PM & PB \\
\hline NB & NB & NB & NS & Z & PS & PM & PB \\
\hline NM & NB & NB & NS & PS & PM & PM & PB \\
\hline NS & NB & NB & NS & PS & PM & PB & PB \\
\hline Z & NB & NB & Z & PM & PB & PM & PB \\
\hline PS & NB & NB & NS & PS & PM & PM & PB \\
\hline PM & NB & NB & NS & PS & PM & PM & PB \\
\hline PB & NB & NB & NS & $Z$ & PS & PM & PB \\
\hline
\end{tabular}

According to Table 2, we can get surface figure of variable transmission ratio: 


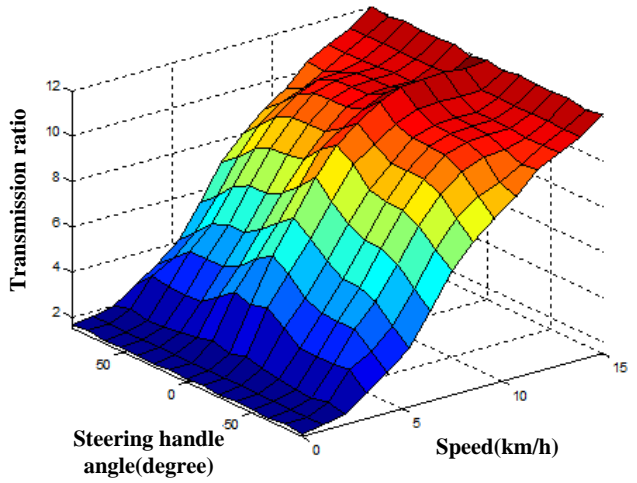

Fig.6. Surface figure of variable transmission ratio

\section{Simulation and Analysis}

Fig.7 indicates the sinusoidal signal of the steering handle angle at the speed of $5 \mathrm{~km} / \mathrm{h}$. Fig. 8 shows the steering wheel angle responses in different steering systems at the speed of $5 \mathrm{~km} / \mathrm{h}$. From Fig. 8 we can see that MS system's maximum steering wheel angle is only $3.72^{\circ}$ (transmission ratio is fixed at 8 ) when the steering handle angle is $\max \left(30^{\circ}\right)$. While SBW system's steering wheel angle is $5.4^{\circ}$ at the same situation, 1.45 times of the MS system. It indicates that the transmission ratio based on fuzzy variable transmission ratio control has benefits to improve the forklift's steering sensitivity at a low speed.

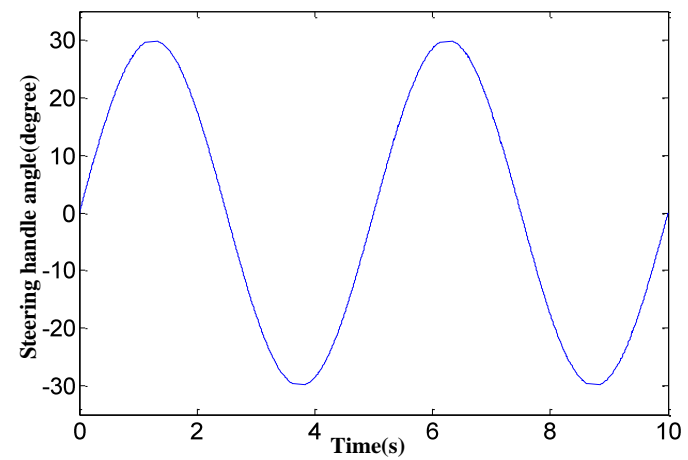

Fig.7. Steering handle angle at speed of $5 \mathrm{~km} / \mathrm{h}$

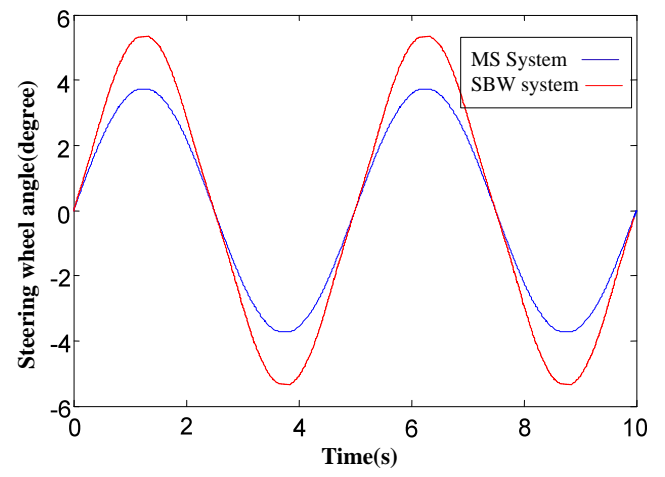

Fig.8. Steering wheel angle in different steering systems at speed of $5 \mathrm{~km} / \mathrm{h}$

Fig. 9 shows the sinusoidal signal of the steering handle angle at speed of $14 \mathrm{~km} / \mathrm{h}$. Fig. 10 shows the steering wheel angle responses in different steering systems at speed of $14 \mathrm{~km} / \mathrm{h}$. From Fig. 10 we can see that MS system's maximum steering wheel angle is $1.25^{\circ}$ (transmission ratio is fixed at 8 ) when the steering handle angle is $\max \left(10^{\circ}\right)$. While SBW system's steering wheel angle is $0.86^{\circ}$ at the same situation, 0.69 times of the MS system. It indicates that the transmission ratio based on fuzzy variable transmission ratio control has benefits to improve the forklift's steering stability at a high speed.

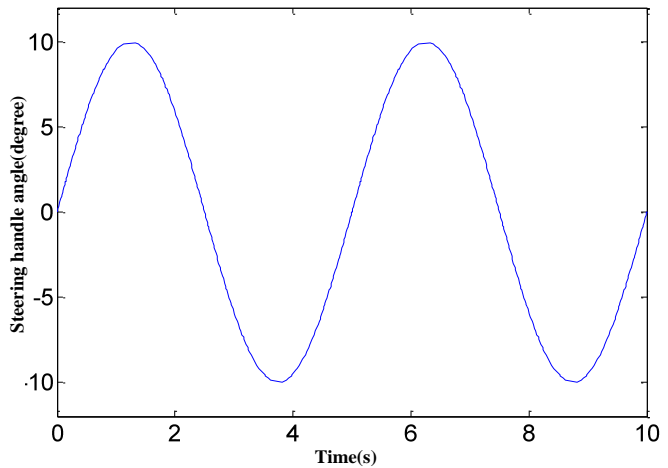

Fig.9. Steering handle angle at speed of $14 \mathrm{~km} / \mathrm{h}$

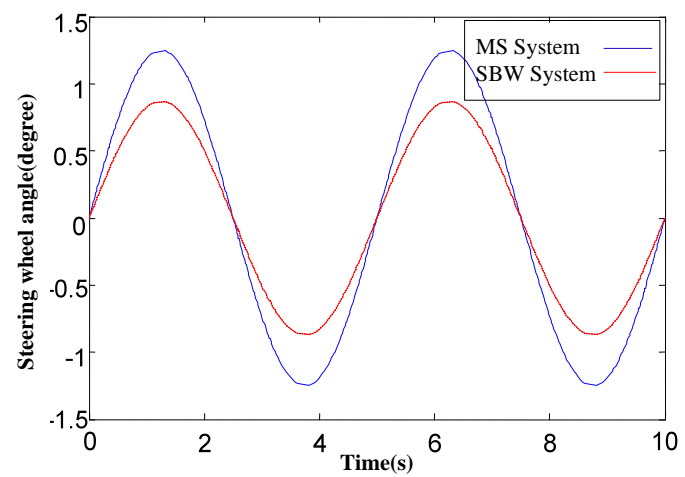

Fig.10. Steering wheel angle in different steering systems at speed of $14 \mathrm{~km} / \mathrm{h}$

From the analysis we can see that the fuzzy variable transmission ratio control can make forklift steering sensitive at low-speed and steering steady at high-speed, and also can improve the operation stability and reduce the driver's load.

\section{THE STUdY ON SBW System's YaW RATE FEEDBACK CONTROL STRATGEY}

\section{A. The Relationship Between Yaw Rate and Forklift Handling Stability}

The main factors influencing the forklift's motion stability are the yaw rate and sideslip angle [27-29]. They reflect the essential characteristics of the forklift's steering movement. The sideslip angle reflects the forklift's track keeping, and indicates the trajectory deviation and lateral acceleration during steering. The yaw rate shows the forklift's understeer or oversteer character, and e mphasizes forklift's operation stability. When the forklift steers normally, the sideslip angle is relatively small, thus 
the paper mainly studies the relationship between yaw rate and operation stability.

The forklift's heading angle $(\theta)$ is the sum of the sideslip angle $(\beta)$ and yaw angle $(\psi)$ :

$$
\theta=\beta+\psi=\beta+\int \omega_{\gamma} \mathrm{dt}
$$

When we ignore the sideslip angle, the heading angle basically depends on the yaw angle. And the yaw angle depends on the yaw rate. Thus the yaw rate reflects the forklift's steering characters. The larger the yaw rate becomes, the bigger the heading angle is, and the smaller the turn radius is. The opposite is the other way around. Now we analyze the relationship between the yaw rate and steering characters to reflect the relationship between yaw rate and forklift operation stability.

The main parameter to evaluate the forklift's stationary response is the difference between front wheel sideslip angle $\left(\alpha_{1}\right)$ and back wheel sideslip angle $\left(\alpha_{2}\right)$ :

$$
\alpha_{1}-\alpha_{2}=\left\{\begin{array}{c}
<0, \text { understeer } \\
0, \text { neutralsteer } \\
>0, \text { oversteer }
\end{array}\right.
$$

We can get the relationship between $\alpha_{1}$ and $\alpha_{2}$ according to velocity composition law:

$$
\alpha_{1}-\alpha_{2}=\frac{L \omega_{\gamma}}{u}-\delta_{w h}
$$

Bring (9) into (8), we can get:

$$
\omega_{\gamma}=\left\{\begin{array}{c}
<\frac{u \delta_{w h}}{L}, \text { understeer } \\
\frac{u \delta_{w h}}{L}, \quad \text { neutralsteer } \\
<\frac{u \delta_{w h}}{L}, \text { oversteer }
\end{array}\right.
$$

From (10) we can get the forklift's steering characters to judge the forklift's stability situation by analyzing the yaw rate.

\section{B. The Research of The Yaw Rate Feedback Control Strategy}

Though the fuzzy variable transmission ratio based on the steering handle angle and vehicle speed is established on the forklift's dynamic response, the design of steering transmission ratio function doesn't consider the influence of the yaw rate. The practical yaw rate is changing when the forklift steers at a narrow space frequently. If the yaw rate changes too fast, the forklift's handling stability must be influenced directly $[30,31]$. Thus this paper adds the yaw rate feedback control based on the fuzzy variable transmission ratio control. So the system can make dynamic compensation of practical steering wheel angle to improve the forklift's dynamic characteristics.

The yaw rate feedback control is showed in Fig.11. When the forklift's steering state changes, we can get the difference between the desired yaw rate $\left(\omega_{\gamma}^{*}\right)$ calculated by fuzzy variable transmission ratio controller and the practical yaw rate $\left(\omega_{\gamma}\right)$, and enter the difference into the controller. The controller can get the front wheel compensation angle $(\Delta \delta)$ by calculation. Then we can get the targeted steering wheel angle $\left(\omega_{w h}^{*}\right)$ by calculating the summation of steering wheel angle $(\delta)$ got from fuzzy variable transmission ratio controller and $\Delta \delta$. Next the $\omega_{\mathrm{wh}}^{*}$ is entered into the main controller. The main controller controls the motor to realize the forklift's steering control.

If we want to realize forklift's SBW system's yaw rate feedback control, firstly we should get the desired yaw rate. When the forklift steers under the normal situation, in order to maintain the driver's driving habits and improve steering sensitivity, we generally take the steady yaw rate obtained from linear two degree of freedom model as the desired yaw rate. Then we can get:

$$
\omega_{\gamma}=\frac{\frac{u}{L}}{i\left(1+\frac{m}{L^{2}}\left(\frac{a}{k_{2}}-\frac{b}{k_{1}}\right) u^{2}\right)} \delta_{s w}
$$

To ensure the security and stability of the forklifts under extreme driving conditions, the forklift's tire adhesion limits on different roads should be considered. Thus the lateral acceleration should meet the following criteria:

$$
\left|a_{y}\right| \leq \mu g
$$

Where $g$ represents the gravitational acceleration and $\mu$ denotes the road adhesion coefficient. When $\beta$ is relatively small, we can approximately get $a_{y}$ :

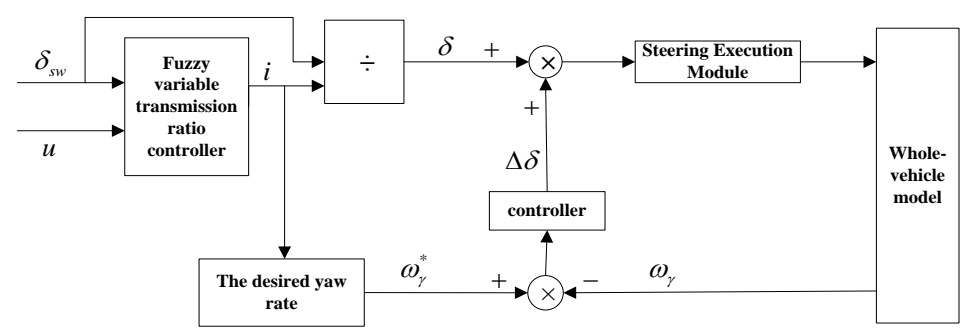

Fig.11. The control block of yaw rate feedback 


$$
a_{y} \approx \frac{u^{2}}{R}=\omega_{\gamma} u
$$

Where $\mathrm{R}$ means the turning radius

According to (11) (13), the desired yaw $\operatorname{rate}\left(\omega_{\gamma}^{*}\right)$ is described as follows:

$$
\omega_{\gamma}^{*}=\min \left\{\left|\frac{u \delta_{s w}}{L\left(1+\frac{m}{L^{2}}\left(\frac{a}{k_{2}}-\frac{b}{k_{1}}\right) u^{2}\right)}\right|,\left|\frac{\mu g}{u}\right|\right\} \operatorname{sgn}\left(\delta_{s w}\right)
$$

Then SBW system can implement the yaw rate feedback control to make the steering wheel get suitable steering angle to realize the active steering.

\section{The Yaw Rate Feedback Based on Fuzzy Self-adaptive PID Control}

Through the above analysis we can get that the yaw rate is an important variable to evaluate the forklift's handling stability. We take the desired yaw rate as the control objective in this paper. Combined with the fuzzy control's characteristics of strong robustness, high PID control accuracy and good stability, this paper designs the fuzzy self-adaptive PID controller to realize the quick and accurate tracking control of the desired yaw rate and to improve forklift's dynamic property.

\section{1) The structure of fuzzy self-adaptive PID controller}

As is shown in the Fig.12, fuzzy controller and PID controller together make up the fuzzy self-adaptive PID controller. Fuzzy controller's input variables are the deviation $(e)$ between the practical yaw $\operatorname{rate}\left(\omega_{\gamma}\right)$ and desired yaw rate $\left(\omega_{\gamma}^{*}\right)$ and deviating rate $(e c)$. Fuzzy control's output variables are proportionality coefficient deviation $\left(\Delta k_{p}\right)$, integral coefficient deviation $\left(\Delta k_{i}\right)$ and differential coefficient deviation $\left(\Delta k_{d}\right)$. The fuzzy controller can adjust $\Delta k_{p}, \Delta k_{i}$, and $\Delta k_{d}$ online according to $e, e c$ and fuzzy control rules. Then the fuzzy controller can adjust the PID controller's proportionality coeffi$\operatorname{cient}\left(k_{d}\right)$, integral coefficient $\left(k_{i}\right)$ and differential coeffi$\operatorname{cient}\left(k_{d}\right)$ to realize the different $e$ and $e c$ corresponding different control parameters, improve the forklift's dynamic response and complete steady steering control.

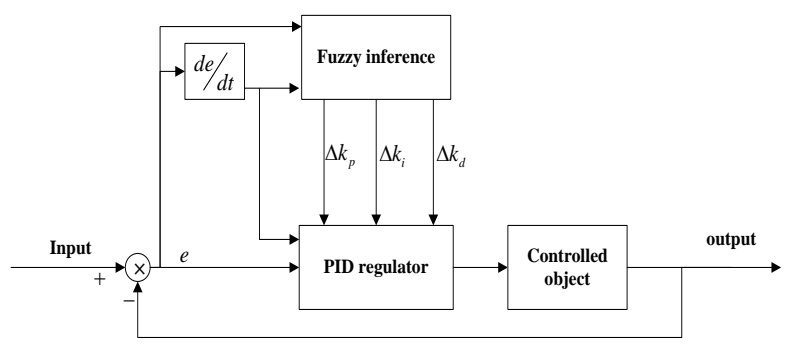

Fig.12. The structure diagram of fuzzy adaptive PID controller

\section{2) The design of fuzzy self-adaptive PID controller}

\section{The linguistic variables and fuzzy discourse domains} of input and output

The linguistic variables of input and output are both divided into seven degrees, namely Negative $\mathrm{Big}(\mathrm{NB})$, Negative Middle(NM), Negative Small(NS), Zero(Z), Positive Small(PS), Positive Middle(PM), Positive $\mathrm{Big}(\mathrm{PB})$. Because of the steering tires' self-righting effect, the forklift's motion state is relatively steady when the forklift steers normally. Thus there isn't a big difference between the car's practical yaw rate and desired yaw rate. So the input variables yaw $\operatorname{rate}\left(\omega_{\gamma}^{*}\right)$ 's deviation(e) and deviating rate(ec)'s fuzzy discourse domain is designed from -1.2 to 1.2. $\Delta k_{p}$ 's fuzzy discourse domain is designed from -3 to $3 . \Delta k_{i}$ 's fuzzy discourse domain is designed from -0.6 to 0.6 . $\Delta k_{d}$ 's fuzzy discourse domain is designed from -3 to 3 .

2. The membership functions of input and output variables

The input and output's membership functions are both triangular membership function. As are shown from Fig.13 to Fig.15.

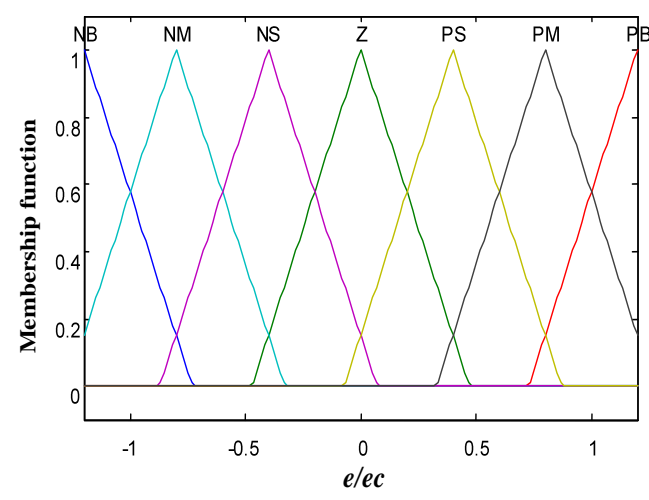

Fig.13. Membership function chart of $e / e c$

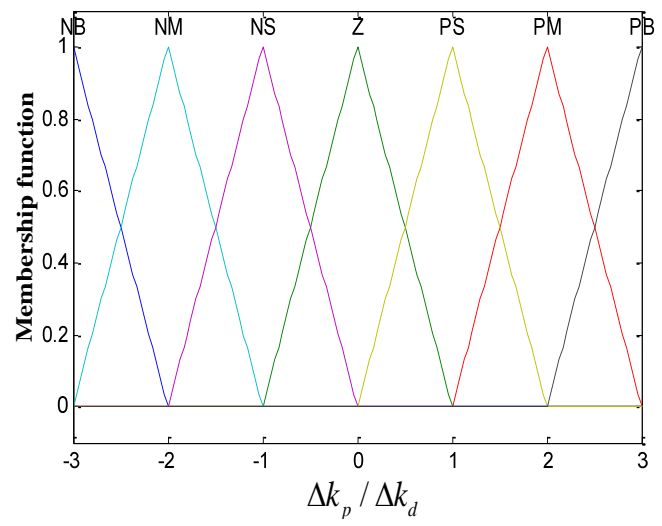

Fig.14. Membership function chart of $\Delta k_{p} / \Delta k_{d}$ 


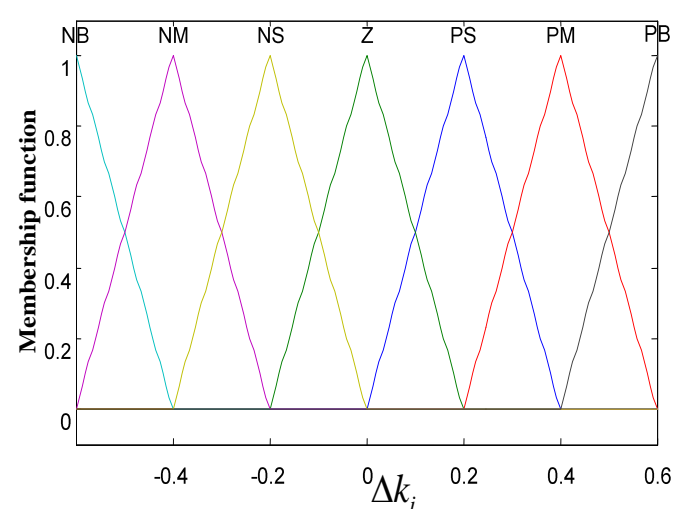

Fig.15. Membership function chart of $\Delta k_{i}$

\section{Fuzzy control rules}

PID controller's parameters are usually designed according to the relationships and effects of $k_{p}, k_{i}$ and $k_{d}$. While the fuzzy self-adaptive PID control calculates $e$ and $e c$ based on PID control in real time. According to the fuzzy control rules to do fuzzy inference, we can get corresponding parameters by looking up tables. The fuzzy control rules of $\Delta k_{p}, \Delta k_{i}, \Delta k_{d}$ are shown in Table 3 to Table 5 .

Table 3. The fuzzy control rule table of $\Delta k_{p}$

\begin{tabular}{|c|c|c|c|c|c|c|c|}
\hline \multirow{2}{*}{$e$} & \multicolumn{7}{|c|}{$e c$} \\
\cline { 2 - 8 } & NB & NM & NS & Z & PS & PM & PB \\
\hline NB & PB & PB & PM & PM & PS & Z & Z \\
\hline NM & PB & PB & PM & PS & PS & Z & NS \\
\hline NS & PM & PM & PM & PS & Z & NS & NS \\
\hline Z & PM & PM & PS & Z & NS & NM & NM \\
\hline PS & PS & PS & Z & NS & NS & NM & NM \\
\hline PM & PS & $Z$ & NS & NM & NM & NM & NB \\
\hline PB & Z & $Z$ & NM & NM & NM & NB & NB \\
\hline
\end{tabular}

Table 4. The fuzzy control rule table of $\Delta k_{i}$

\begin{tabular}{|c|c|c|c|c|c|c|c|}
\hline \multirow{2}{*}{$e$} & \multicolumn{7}{|c|}{$e c$} \\
\cline { 2 - 8 } & NB & NM & NS & Z & PS & PM & PB \\
\hline NB & NB & NB & NM & NM & NS & Z & $Z$ \\
\hline NM & NB & NB & NM & NS & NS & Z & Z \\
\hline NS & NB & NM & NS & NS & Z & PS & PS \\
\hline Z & NM & NM & PS & Z & PS & PM & PM \\
\hline PS & NM & NS & Z & PS & PS & PM & PB \\
\hline PM & Z & $Z$ & PS & PS & PM & PB & PB \\
\hline PB & $Z$ & $Z$ & PS & PM & PM & PB & PB \\
\hline
\end{tabular}

Table 5. The fuzzy control rule table of $\Delta k_{d}$

\begin{tabular}{|c|c|c|c|c|c|c|c|}
\hline \multirow{2}{*}{$e$} & \multicolumn{7}{|c|}{$e c$} \\
\cline { 2 - 8 } & NB & NM & NS & Z & PS & PM & PB \\
\hline NB & PS & NS & NB & NB & NB & NM & PS \\
\hline NM & PS & NS & NB & NM & NM & NS & Z \\
\hline NS & Z & NS & NM & NM & NS & NS & Z \\
\hline Z & Z & NS & NS & NS & NS & NS & Z \\
\hline PS & Z & Z & Z & Z & Z & Z & Z \\
\hline PM & PB & NS & PS & PS & PS & PS & PB \\
\hline PB & PB & PM & PM & PM & PS & PS & PB \\
\hline
\end{tabular}

4. Fuzzy inference and defuzzification

After we confirm the fuzzy rules, we still need to do fuzzy inference to get PID's three parameters' fuzzy subsets. This paper takes widely used Mamdani method to do fuzzy inference. It is a complex fuzzy inference method and takes the "maximum-minimum" rule to describe the meaning of fuzzy inference.

The fuzzy quantities got through fuzzy inference can't be used as the output. They must be transformed into exact values to be entered into the control actuator. This process is called defuzzification. This paper uses gravity method to do the defuzzification.

\section{The Results and Analysis of Simulation}

When the road adhesion coefficient $(\mu)$ is 0.5 , the speed are $3 \mathrm{~km} / \mathrm{h}, 7 \mathrm{~km} / \mathrm{h}$ and $14 \mathrm{~km} / \mathrm{h}$, and the steering handle angle is the step signal of $30^{\circ}$, the yaw rate step responses of the traditional mechanical steering control (transmission ratio is fixed as 8), non-feedback fuzzy variable transmission ratio control and yaw rate feedback fuzzy variable transmission ratio control are shown on the Fig.16 to Fig.18.

From Fig. 16 to Fig.18 we can see that when the forklift steers at a low speed, the traditional mechanical steering control's yaw rate steady value is smaller than the desired yaw rate. It means that the forklift presents the character of understeer, the forklift steers dully, and the driver feels difficult to turn the car. When the forklift steers at a high speed, the traditional mechanical steering control's yaw rate steady value is bigger than the desired yaw rate. It means that the forklift presents the character of oversteer, and the forklift steers sensitively. The car's stability is not good at a high speed. The yaw rate steady value of non-feedback fuzzy variable trans mission ratio control and yaw rate feedback fuzzy variable transmission ratio control both can follow the desired yaw rate accurately and quickly. It means that the operation stability is good under the full speed situation. And the desired yaw rate is closed to the traditional mechanical steering control's yaw rate steady value at the middle speed. It indicates the steering character is similar to the traditional mechanical steering system. Thus the driver has no need to change their driver habit and has a good adaptation to the steering. 


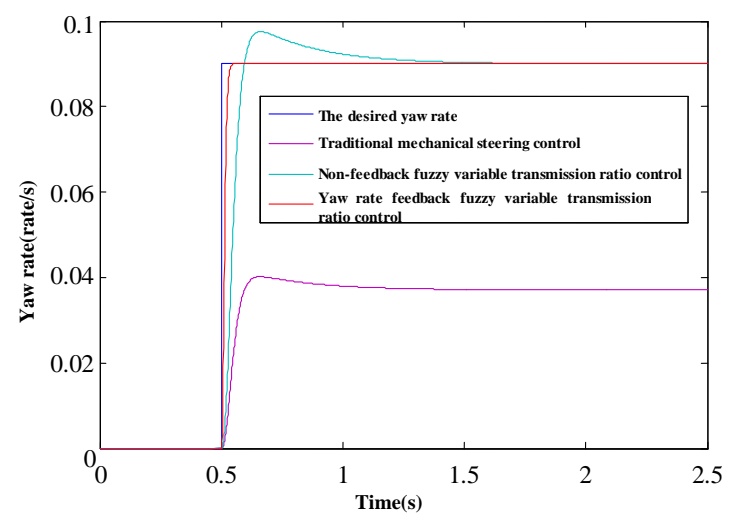

Fig.16. Yaw rate step response at speed of $3 \mathrm{~km} / \mathrm{h}$

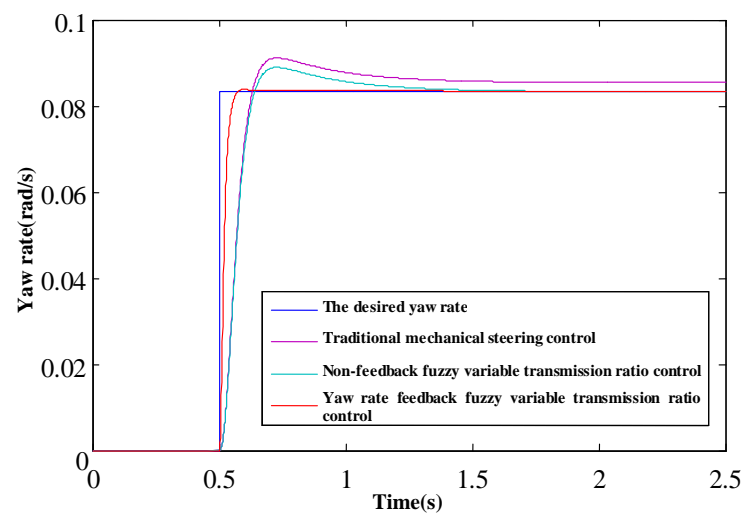

Fig.17. Yaw rate step response at speed of $7 \mathrm{~km} / \mathrm{h}$

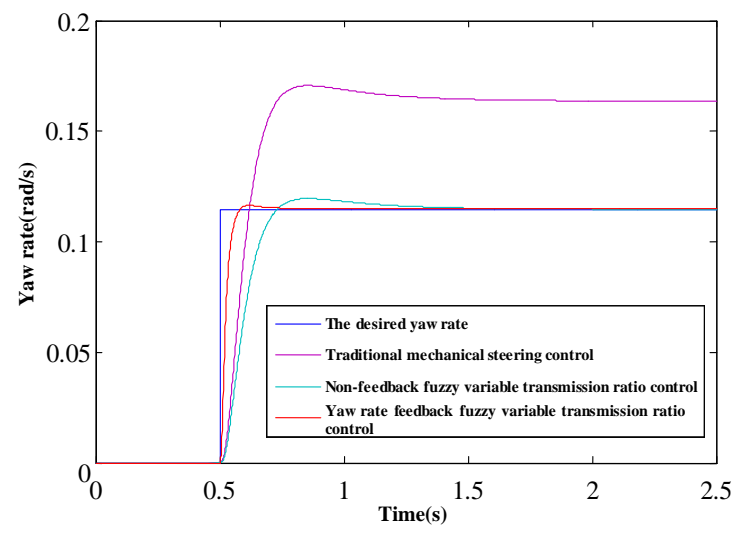

Fig.18. Yaw rate step response at speed of $14 \mathrm{~km} / \mathrm{h}$

\section{CONCLUSIONS}

As an industrial truck widely used under complex conditions such as warehouses, workshops, docks and so on, electric forklifts can improve operating efficiency and reduce labor intensity. Since electric forklift steers frequently in a narrow space and has obvious load variations, performance requirements for forklift's steering system are higher than ordinary vehicles. It requires not only the steering sensitivity, but also good operation stability. Traditional mechanical and hydraulic steering system can't completely solve the light and sensitivity con- tradiction of steering. As a new electronically controlled steering system, SBW system gets rid of the shackles of mechanical or hydraulic structure, and the design of vehicle steering characteristics is free. So SBW system is the future development direction of the forklift steering system.

Through the analysis of the steering system's transmission ratio characteristics and the introduce of the ideal transmission ratio's concept, in this paper, we design the ideal transmission ratio based on constant yaw rate gain according to the parameters of TE60. On the basic of analy zing the factors affecting the trans mission ratio, we establish a fuzzy variable transmission ratio control method based on steering handle angle and vehicle speed. The simulation results show that fuzzy variable transmission ratio control method has obviously improved the steering sensitivity at a low speed and the steering stability at the high speed, enhancing the forklift's handling performance.

This paper analyzes the relationship between the yaw rate and operation stability, proposes the yaw rate feedback control based on the fuzzy variable transmission ratio control, and designs a fuzzy self-adaptive PID controller. The simulation results show that after introducing the yaw rate feedback control, the forklift's practical yaw rate at low, medium and high speed is able to quickly and accurately track the desired yaw rate, shortening the forklift's response time and improving the tracking performance.

\section{REFERENCES}

[1] Avinash Balachandran, J.Christian Gerdes , "Designing Steering Feel for Steer-by-Wire Vehicles Using Objective Measures", IEEE/ASME Transactions on Mechatronics, Vol.20, No.1, pp. 373-383,2015.

[2] Cristian Chitu, Jochen Lackner, Martin Horn, et al. "Controller design for an electric power steering system based on LQR techniques", The International Journal for Computation and Mathematics in Electrical and Electronic Engineering, Vol. 32, No. 3, pp. 763-775,2013.

[3] C. Dannöhl, S.Müller, H.Ulbrich, "Hळ-control of a rack-assisted electric power steering system", Vehicle System Dynamics, Vol. 50, No. 4, pp. 527-544, 2012.

[4] Farbod Fahimi. "Full drive-by-wire dy namic control for four-wheel-steer all-wheel drive vehicles". Vehicle System Dynamics, 51(3): 360-376, 2013.

[5] M. Halton, M. J. Hayes, P. Iordanov, "State-space $\mu$ analysis for an experimental drive-by-wire vehicle". International Journal of Robust and Nonlinear Control, 18(9): 975-992, 2008.

[6] Hai Wang, Zhihong Man, Weixiang Shen, et al, "Robust control for Steer-by-Wire systems with partially known dynamics," IEEE Transactions on Industrial Informatics, Vol. 10, No. 4, pp. 2003-2015,2014.

[7] Y. Marumo and M. Nagai, "Steering control of motorcycles using steer-by-wire system," Veh. Syst. Dyn., vol. 45, no. 9, pp. 445-458, 2007.

[8] Y. Marumo and N. Katagiri, "Control effects of steer-by-wire system for motorcycles on lane-keeping performance," Veh. Syst. Dyn., vol. 49, no. 8, pp. 1283-1298, 2011.

[9] Y. Yamaguchi and T. Murakami, "Adaptive control for virtual steering characteristics on electric vehicle using 
Steer-by-Wire system," IEEE Trans. Ind. Electron., vol. 56, no. 5, pp. 1585-1594, May 2009.

[10] K. Nam, H. Fujimoto, and Y. Hori, "Lateral stability control of in-wheel-motor- driven electric vehicles based on sideslip angle estimation using lateral tire force sensors," IEEE Trans. Veh. Technol., vol. 61, no. 5, pp. 1972-1985, Jun. 2012.

[11] A. E. Cetin, M. A. Adli, and D. E. Barkana, "Implementation and development of an adaptive steering-control system," IEEE Trans. Veh. Technol., vol. 59, no. 1, pp. 75-83, Jan. 2010.

[12] H. Wang, Z. Man, H. Kong, and W. Shen, "Terminal sliding mode control for Steer-by-Wire system in electric vehicles," in Proc. IEEE Conf. Ind. Electron. Appl. (ICIEA), pp.919-924., 2012.

[13] Yousuke Yamaguchi, Toshiyuki Murakami. "Adaptive Control for Virtual Steering Characteristics on Electric Vehicle Using Steer-by-Wire System". IEEE Transactions on Industrial Electronics, Vol. 56, No. 5, pp. 1585-1594, 2009.

[14] Balach andran A, Gerdes J C. Designing Steering Feel for Steer-by-Wire Vehicles Using Objective Measures [J]. IEEE/ASME Transactions on Mechatronics, 20(1):373-383., 2015.

[15] M. Segawa, S. Kimura, T. Kada, and S. Nakano, "A study of reactive torque control for steer by wire system," in Proc. Int. Symp. AVEC, pp. 653-657, 2002.

[16] P. Yih and J. C. Gerdes, "Modification of vehicle handling characteristics via steer-by-wire," IEEE Trans. Control Syst. Technol., vol. 13, no. 6, pp. 965-976, Nov. 2005.

[17] Hongliang Zhou, Zhiyuan Liu, "Vehicle Yaw Stability-Control System Design Based on Sliding Mode and Backstepping Control Approach". IEEE Transactions on Vehicular Technology, Vol. 59, No. 7, pp. 3674-3678, 2010.

[18] Gerry Howser, Bruce McMillin, "Modeling and reasoning about the security of drive-by-wire automobile systems". International Journal of Critical Infrastructure Protection, 5(3-4): 127-134, 2012.

[19] Jin Hur, "Characteristic analysis of interior permanent-magnet synchronous motor in electrohydraulic power steering systems", IEEE Transactions on Industrial Electronics, Vol. 55, No. 6, pp. 2316-2323,2008

[20] Alaa Marouf, Mohamed Djemai, Chouki Sentouch, Philippe Pudlo. "A New Control Strategy of an Electric-Power-Assisted Steering System", IEEE Transactions on Vehicular Technology, Vol. 61, No. 8, pp. 3574-3589,2012.

[21] Manh Tuan Do, Zhihong Man, Cishen Zhang, et al, "Robust sliding mode-based learning control for Steer-by-Wire systems in modern vehicles," IEEE Transactions on Vehicular Technology, Vol. 63, No. 2, pp. 580-590,2014.

[22] Masaya Segawa, Shiro Nakano, Osamu Nishihara, Hiromitsu Kumamoto. "Vehicle stability control strategy for steer by wire system”. JSAE Review, 22(9):383-388, 2001.

[23] Pradeep Setlur, John R. Wagner, Darren M. Dawson, David Braganza. "A Trajectory Tracking Steer-by-Wire Control Sy stem for Ground Vehicles". IEEE Transactions on Vehicular Technology, Vol.55, No.1, pp.76-85, 2006.

[24] P. Lemerle, O. Höppner, J. Rebelle. "Dynamic stability of forklift trucks in cornering situations: parametrical analysis using a driving simulator". Vehicle System Dynamics, 4910, 2011.
[25] Qun Zeng, "The design of power assisted characteristic curve for electric power steering system", Journal of Convergence Information Technology, Vol. 7, No. 17, pp. 260-266,2012.

[26] Mirko Rinchi, Luca Pugi, Fabio Bartolini, and Luigi Gozzi, "Design of control sy stem to prevent forklift capsize", International Journal of Vehicle Systems Modeling and Testing, Vol.5, No.1, pp.35-58,2010.

[27] Tao Yang. "A New Control Framework of Electric Power Steering System Based on Admittance Control". IEEE Transactions on Control Systems Technology, Vol. 23, No. 2, pp. 762-769, 2015.

[28] Tao Meng, Hui Chen, “A Study on the Control Strategy for Wheel Return and Active Damping of Electric Power Steering System", Automotive Engineering, Vol.28, No.12, pp. 1125-1128, 2006.

[29] Xu Xiang, Song Wenbin, "A Discussion on the Application of EPS on Electric Forklift", Journal of Mechanical \& Electrical Engineering, Vol. 27, No. 11, pp. 33-35,64,2010.

[30] Xiang Chen, Tiebao Yang, Xiaoqun Chen, Kemin Zhou, "A generic model-based advanced control of electric power-assisted steering systems", IEEE Transactions on Control Systems Technology, Vol. 16, No. 6, pp. 1289-1300,2008.

[31] Yan He, Benxian Xiao. "Research on Power Characteristic of the Electric Forklift EPS System". International Journal on Smart Sensing and Intelligent Systems, Vol.8, No.3, pp. 1768-1785, 2015.

[32] Zineb LAOUICI, Mohammed Amine MAMI, Mohamed Fayçal KHELFI. "Hybrid Method for the Navigation of Mobile Robot Using Fuzzy Logic and Spiking Neural Networks" International Journal of Intelligent Systems and Applications, Vol. 6, No. 12, pp.1-9,2014.

\section{Authors' Profiles}

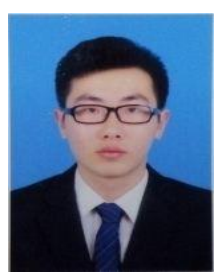

Guohua Xiang received the B.E. degree from the School of Electrical Engineering in Zhengzhou University in 2010. Since 2014, he is working with Benxian Xiao as his student for the Master's degree in control theory and control engineering in Hefei University of Technology, Hefei, China.

His current research interests include steering-by-wire system, the fuzzy self-adaptive PID control, and the variable transmission ratio control.

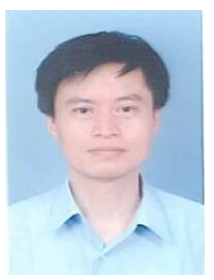

Benxian Xiao was born on April 4, 1964 in Anhui, China. He received the B.Sc degree and the M.Sc degree and Ph.D. degree in Electrical Engineering and Automation from Hefei University of Technology, Hefei, China, in 1986 and 1989 and 2004, respectively. Since 1989, he has been with the Department of Automation, School of Electrical

Engineering and Automation, Hefei University of Technology. Now he is a Professor in Control Theory \& Control Engineering Subjects. His current research interests include Intelligent Control, Automotive Steering Control Systems, System Modeling and Simulation. 